\title{
NECESSARY AND SUFFICIENT CONDITIONS FOR A SPACE TO HAVE A MAXIMAL, PROPER, DENSE, $T_{3 \frac{1}{2}}$ SUBSPACE
}

\section{CHARLES DORSETT}

Department of Mathematics

Texas A\&M University-Commerce

Texas 75429

USA

e-mail: charles.dorsett@tamuc.edu

\begin{abstract}
Within this paper necessary and sufficient conditions for a space to have a maximal, proper, dense, $T_{3 \frac{1}{2}}$ subspace are given and subspaces properties of such spaces are further investigated.
\end{abstract}

\section{Introduction and Preliminaries}

In the 1936 paper [9], for a space $(X, T)$, an externally generated, strongly $(X, T)$ related space, called the $T_{0}$-identification space of $(X, T)$, was introduced and used to jointly characterize metrizable and pseudometrizable.

2010 Mathematics Subject Classification: 54B05, 54D15.

Keywords and phrases: proper subspaces, dense subspaces, maximal $T_{3 \frac{1}{2}}$ subspaces.

Received February 28, 2018

(ㄷ) 2018 Scientific Advances Publishers 
Definition 1.1. Let $(X, T)$ be a space, let $R$ be the equivalence relation on $X$ defined by $x R y$ iff $C l(\{x\})=C l(\{y\})$, let $X_{0}$ be the set of $R$ equivalence classes of $X$, let $N: X \rightarrow X_{0}$ be the natural map, and let $Q(X, T)$ be the decomposition topology on $X_{0}$ determined by $(X, T)$ and the map $N$. Then $\left(X_{0}, Q(X, Y)\right)$ is the $T_{0}$-identification space of $(X, T)$.

Theorem 1.1. A space $(X, T)$ is pseudometrizable iff $\left(X_{0}, Q(X, T)\right)$ is metrizable. $T_{0}$-identification spaces were cleverly created to add $T_{0}$ to the externally generated, strongly $(X, T)$ related $T_{0}$-identification space of $(X, T)$ [9], which, when combined with the fact that metrizable and (pseudometrizable and $T_{0}$ ) are equivalent, was used to establish the result above.

The characterization of metrizable and pseudometrizable given above using $T_{0}$-identification spaces raised the question: What other properties $P$ not necessarily $T_{0}$ and ( $P$ and $T_{0}$ ), if any, would behave in the same manner as pseudometrizable and metrizable $=$ (pseudometrizable and $\left.T_{0}\right)$ ?, which led to the introduction and investigation of weakly $P o$ properties in 2015 [1].

Definition 1.2. Let $P$ be topological properties such that $P o=\left(P\right.$ and $\left.T_{0}\right)$ exists. Then a space $(X, T)$ is weakly $P o$ iff its $T_{0}$-identification space $\left(X_{0}, Q(X, T)\right)$ has property $P$. A topological property $P o$ for which weakly $P o$ exists is called a weakly $P o$ property [1].

Since for a space, its $T_{0}$-identification space has property $T_{0}$, then, for a topological property $P$ for which $P o$ exists, a space is weakly $P o$ iff its $T_{0}$-identification space has property $P o$. 
Within the paper [1], it was shown that for a weakly Po property $Q o$, a space is weakly $Q_{o}$ iff its $T_{0}$-identification space is weakly $Q_{o}$, which led to the introduction and investigation of $T_{0}$-identification $P$ properties [2].

Definition 1.3. Let $S$ be a topological property. Then $S$ is a $T_{0}$-identification $P$ property iff both a space and its $T_{0}$-identification space simultaneously shares property $S$.

In the introductory weakly $P o$ property paper [1], it was shown that weakly $P o$ is neither $T_{0}$ nor "not- $T_{0}$ ", where "not- $T_{0}$ " is the negation of $T_{0}$. The need and use of "not- $T_{0}$ " revealed "not- $T_{0}$ " as a useful, powerful topological property and tool, motivating the inclusion of the long-neglected properties "not- $P$ ", where $P$ is a topological property for which "not- $P$ " exists, as important properties for investigation and use in the study of topology. As a result, within a short time period, many new, important, fundamental, foundational, never before imagined properties and examples have been discovered, expanding and changing the study of topology forever.

In past studies of weakly $P o$ spaces and properties, for a classical topological property $Q o$, a special topological property $W$ was sought such that for a space with property $W$, its $T_{0}$-identification space has property Qo, which then implies the initial space has property $W$, with no certainty that such a topological property $W$ exists. As given above, the study of weakly $P o$ spaces and related properties has been a productive study, but, if the past search process continued, the study of weakly Po spaces and properties would continue to be uncertain, tedious, and never ending. To make the process more certain, the question of precisely which topological properties are weakly $P o$ properties arose leading to answers in a 2017 paper [3]. 
Answer 1.1. Let $Q$ be a topological property for which both $Q o$ and ( $Q$ and "not- $T_{0}$ ") exist. Then $Q$ is a $T_{0}$-identification $P$ property that is weakly $P o$ and $Q=$ weakly $Q o=\left(Q o\right.$ or $\left(Q\right.$ and "not- $T_{0}$ " $\left.)\right)[3]$.

Answer 1.2. $\left\{Q o \mid Q\right.$ is a $T_{0}$-identification $P$ property $\}=\{Q o \mid Q o$ is a weakly $P o$ property $\}=\{Q o \mid Q$ is a topological property and $Q o$ exists $\}$ [3].

Thus, major progress was achieved in the study of topology, weakly $P o$, and related properties. If $Q$ is a topological property for which both $Q o$ and $\left(Q\right.$ and "not- $T_{0}$ ") exist, Answer 1.1 quickly and easily gives weakly $Q o$. If $Q$ is a topological property for which $Q=Q o$, then $Q=Q o$ is a weakly $P o$ property, but $Q=Q o$ is not a $T_{0}$-identification $P$ property or weakly Po. Within the paper [3], a topological property $W$ that can be both $T_{0}$ and "not- $T_{0}$ " was shown to exist that is a $T_{0}$-identification $P$ property that is weakly $P_{0}$ such that $W=$ weakly $Q o$, again making the search process certain, but, just knowing such a $W$ exists, gave little insight into the precise, needed topological property $W$, raising the question of whether the known information could somehow be used to more precisely determine $W$ for the fixed $Q o$.

The investigation of that question led to the introduction and investigation of OXTO subsets and the corresponding subspace for each space $(X, T)[4]$.

Definition 1.4. Let $(X, T)$ be a space and for each $x \in X$, let $C_{x}$ be the $T_{0}$-identification space equivalence class containing $x$. Then $Y$ is an OXTO subset of $X$ iff $Y$ contains exactly one element from each equivalence class $C_{x}$. 
Within the paper [4], it was shown that for a space $(X, T)$, for each OXTO subset $Y$ of $X,\left(Y, T_{Y}\right)$ is homeomorphic to $\left(X_{0}, Q(X, T)\right)$. Since, as stated earlier, the $T_{0}$-identification space of each space is $T_{0}$ and $T_{0}$ is a topological property, then for each $O X T O$ subset $Y$ of $X$ in a space $(X, T),\left(Y, T_{Y}\right)$ is $T_{0}$.

Also, within the paper [4], it was shown that for each topological property $Q$ for which $Q o$ exists, a space $(X, T)$ is weakly $Q o$ iff for each OXTO subset $Y$ of $(X, T),\left(Y, T_{Y}\right)$ has property $Q o$, which can be, and has been, used to precisely determine weakly Qo [4]. Thus, the study of weakly $P o$ and weakly $P o$ properties has been completely internalized and greatly simplified by the use of OXTO subsets and the corresponding subspaces, and the earlier results, replacing many, earlier uncertainties with certainties.

The continued investigation of weakly $P o$ properties, "not- $T_{0}$ ", and OXTO subsets and subspaces of a space led to the definition and unexpected results below.

Theorem 1.2. Let $(X, T)$ be a space. Then the following are equivalent: (a) $(X, T)$ is "not- $T_{0}$ ", (b) $(X, T)$ has a maximal, proper, dense $T_{0}$ subspace, and (c) for each OXTO subset $Y$ of $X,\left(Y, T_{Y}\right)$ is a maximal, proper, dense $T_{0}$ subspace of $(X, T)[5]$.

Definition 1.5. Let $(X, T)$ be a space, let $Y$ be a subspace of $(X, T)$, and let $Q$ be a topological property for which $Q o$ exists. Then $\left(Y, T_{Y}\right)$ is a maximal, proper, dense, $Q o$ subspace of $(X, T)$ iff $\left(Y, T_{Y}\right)$ is a proper, dense, Qo subspace of $(X, T)$ such that for each subspace $\left(Z, T_{W}\right)$ of $(X, T)$, where $Z$ properly containing $Y,\left(Z, T_{Z}\right)$ is "not-Qo" [6]. 
Theorem 1.3. Let $(X, T)$ be a space and let $Q$ be a topological property for which Qo exists. Then (a) for each OXTO subset $Y$ of $X,\left(Y, T_{Y}\right)$ is a maximal, proper, dense, Qo subspace of $(X, T)$ iff (b) $(X, T)$ is weakly Qo and "not-T," [6].

The results above raised questions about necessary and sufficient conditions, if any, for a space to have a maximal, proper, dense, $T_{i}$ subspace, $i=1,2$, which were resolved in the paper [7], raising the question of necessary and sufficient conditions, if any, for a space to have a maximal, proper, dense, $T_{3 \frac{1}{2}}$ subspace.

The completely regular and $T_{3 \frac{1}{2}}$ separation axioms were introduced in 1925 [10].

Definition 1.6. A space $(X, T)$ is completely regular iff for each closed set $C$ and each $x \notin C$, there exists a continuous function $f:(X, T) \rightarrow([0,1], U)$, where $U$ is the usual relative metric topology on $[0,1]$, such that $f(x)=0$ and $f(C)=1$. A completely regular $T_{1}$ space is denoted by $T_{3 \frac{1}{2}}$.

The work in this paper focuses attention on "not- $T_{3 \frac{1}{2}}$ ", whose definition is given below.

Definition 1.7. A space is "not- $T_{3 \frac{1}{2}}$ " iff it is "not- $T_{1}$ " or "notcompletely regular".

In the 2017 paper [8], it was shown that $T_{3 \frac{1}{2}}$ is a weakly Po property with completely regular $=$ weakly $($ completely regular $) o=$ weakly $T_{3 \frac{1}{2}}$. Below Theorem 1.3 is applied to completely regular and $T_{3 \frac{1}{2}}$, giving 
necessary and sufficient conditions in "not- $T_{0}$ " spaces for a space to have a maximal, proper, dense, $T_{3 \frac{1}{2}}$ subspace, and the question above for maximal, proper, dense, $T_{3 \frac{1}{2}}$ subspaces is addressed.

\section{Weakly $T_{3 \frac{1}{2}}$ and "not- $T_{0}$ " Spaces and Necessary and Sufficient Conditions on a Space for the Space to Have a Maximal, Proper, Dense, $T_{3 \frac{1}{2}}$ Subspace}

Corollary 2.1. Let $(X, T)$ be a space. Then (a) for each OXTO subset $Y$ of $X,\left(Y, T_{Y}\right)$ is a maximal, proper, dense, $T_{3 \frac{1}{2}}$ subspace of $(X, T)$ iff (b) $(X, T)$ is (completely regular = weakly (completely regular)o) and "not- $T_{0}$ ".

Theorem 2.1. Let $(X, T)$ be a space and let $Y$ be a proper subset of $X$. Then the following are equivalent: (a) $(X, T)$ has a maximal, proper, dense, $T_{3 \frac{1}{2}}$ subspace $\left(Y, T_{Y}\right),(\mathrm{b})(X, T)$ is "not-T $T_{3 \frac{1}{2}}$ ”, $\left(Y, T_{Y}\right)$ is a proper, $T_{3 \frac{1}{2}}$ subspace of $(X, T)$, and for each $x \in X \backslash Y, W=(\{x\} \cup Y)$, and $C_{W}(x)=\left\{y \in W \mid C l_{W}(\{x\})=C l_{W}(\{y\})\right\}$, every T-open set containing $x$ intersects $Y, C_{W}(x)$ contains at most two elements, and if $C_{W}(x)=\{x\}$ and $\{x\}$ is not $T_{W}$-closed, $\left(W, T_{W}\right)$ is "not- $T_{1}$ ", (or $\left(\{x\}\right.$ is $T_{W}$-closed, $\left(W, T_{W}\right)$ is $T_{1}$, and there exists a $T_{W}$-closed set $C$ and $a z \in(W \backslash C)$ such that there does not exist a continuous function $h:\left(W, T_{W}\right) \rightarrow([0,1], U)$ such that $h(z)=0$ and $h(C)=1$, and $(c)$ $\left(Y, T_{Y}\right)$ is a proper, $T_{3 \frac{1}{2}}$ subspace of $(X, T)$, and for each $x \in X \backslash Y, W=(\{x\} \cup Y)$, and $C_{W}(x)=\left\{y \in W \mid C l_{W}(\{x\})=C l_{W}(\{y\})\right\}$, every T-open set containing $x$ intersects $Y, C_{W}(x)$ contains at most two elements, and if $C_{W}(x)=\{x\}$ and $\{x\}$ is not $T_{W}$-closed, $\left(W, T_{W}\right)$ is 
"not- $T_{0}$ ", $\left(\operatorname{or}\left(\{x\}\right.\right.$ is $T_{W}$-closed, $\left(W, T_{W}\right)$ is $T_{1}$, and there exists a $T_{W}$-closed set $C$ and a $z \in(W \backslash C)$ such that there does not exist a continuous function $h:\left(W, T_{W}\right) \rightarrow([0,1], U)$ such that $h(z)=0$ and $h(C)=1$.

Proof. (a) implies (b): Since $\left(Y, T_{Y}\right)$ is a maximal, proper, $T_{3 \frac{1}{2}}$ subspace of $(X, T)$, then, by definition, $(X, T)$ is "not- $T_{3 \frac{1}{2}}$ ", and $\left(Y, T_{Y}\right)$ is a proper, $T_{3 \frac{1}{2}}$ subspace of $(X, T)$. If $\{x\}$ is $T_{W}$-open, then there exists a $T$-open set $O$ containing $x$ such that $O \cap Y=\phi$ and $\left(Y, T_{Y}\right)$ is not dense in $(X, T)$. Thus $\{x\}$ is not $T_{W}$-open. Suppose $C_{W}(x)$ contains three or more elements. Let $x, a$, and $b$ be distinct elements of $C_{W}(x)$. Then $C l_{W}(\{a\})=C l_{W}(\{x\})=C l_{W}(\{b\})$. Thus $C l_{Y}(\{a\})$ $=C l_{Y}(\{b\})$ and $\left(Y, T_{Y}\right)$ is "not- $T_{0}$ " and hence "not- $T_{1}$ ", which is a contradiction. Suppose $C_{W}(x)=\{x, a\}, a \neq x$. Then $C l_{W}(\{x\})=C l_{W}(\{a\})$ and $\left(W, T_{W}\right)$ is "not- $T_{0}$ ", which implies $\left(W, T_{W}\right)$ is "not- $T_{1}$ " and thus, "not- $T_{3 \frac{1}{2}}$ ". Suppose $C_{W}(x)=\{x\}$ and $\{x\}$ is not $T_{W}$-closed. Then (there exists a $y \in Y$ such $y \in C l_{W}(\{x\})$ and $x \notin C l_{W}(\{y\})$ or $y \notin C l_{W}(\{x\})$ and $\left.x \in C l_{W}(\{y\})\right)$ or for all $y \in Y, x \notin C l_{W}(\{y\})$ and $y \notin C l_{W}(\{x\})$. In either of the first two cases, $\left(W, T_{W}\right)$ is "not- $T_{1}$ " and, thus, $\left(W, T_{W}\right)$ is "not$T_{3 \frac{1}{2}}$ ". Thus, consider the third case. Since for each $y \in Y, y \notin C l_{W}(\{x\})$, then $C l_{W}(\{x\})=\{x\}$. Thus $Y=W \backslash\{x\}$ is $T_{W^{-}}, T_{Y^{-o p e n}}$ and each $T_{Y}$-open set is $T_{W}$-open. Since $\left(Y, T_{Y}\right)$ is $T_{1}, x \notin C l_{W}(\{y\})$ for all $y \in Y$, and $y \notin C l_{W}(\{x\})$ for all $y \in Y$, then $\left(W, T_{W}\right)$ is $T_{1}$. Since $\left(W, T_{W}\right)$ is "not- $T_{3 \frac{1}{2}}$ " and $T_{1}$, then $\left(W, T_{W}\right)$ is "not-completely regular" and there exists a $T_{W}$-closed set $C$ and a $z \notin C$ such that there does not exist a continuous function $f:\left(W, T_{W}\right) \rightarrow([0,1], U)$, where $U$ is the usual relative metric topology on $[0,1]$, such that $f(z)=0$ and $f(C)=1$. 
Clearly (b) implies (c).

(c) implies (a): Since for each $x \in X \backslash Y$, every $T$-open set containing $x$ intersects $Y$, then $\left(Y, T_{Y}\right)$ is dense in $(X, T)$, and $\left(Y, T_{Y}\right)$ is a proper, dense, $T_{3 \frac{1}{2}}$ subspace of $(X, T)$. If $C_{W}(x)=\{x, a\}, a \neq x$, then $C l_{W}(\{x\})=C l_{W}(\{a\})$ and $\left(W, T_{W}\right)$ is "not- $T_{0}$ " and, thus "not- $T_{1}$ " and "not- $T_{3 \frac{1}{2}}$ ". If $\mathrm{C}_{\mathrm{W}}(x)=\{x\}$ and $\{x\}$ is not $T_{W}$-closed, $\left(W, T_{W}\right)$ is "not- $T_{1}$ " and, thus, "not- $T_{3 \frac{1}{2}}$ ", and if $\{x\}$ is $T_{W}$-closed, $\left(W, T_{W}\right)$ is $T_{1}$, and there exists a $T_{W}$-closed set $C$ and a $z \in(W \backslash C)$ such that there does not exist a continuous function $h:\left(W, T_{W}\right) \rightarrow([0,1], U)$ such that $h(z)=0$ and $h(C)=1$, which implies $\left(W, T_{W}\right)$ is "not- $T_{3 \frac{1}{2}}$ ".

\section{An Additional Characterization and Properties of Such Spaces}

Theorem 3.1. Let $(X, T)$ be a space and let $Y$ be a proper subset of $X$. Then (a) $\left(Y, T_{Y}\right)$ is a maximal, proper, dense, $T_{3 \frac{1}{2}}$ subspace of $(X, T)$ iff (b) for each subset $Z$ of $X$ that properly contains $Y,\left(Z, T_{Z}\right)$ is "not- $T_{3 \frac{1}{2}}$ ", and each subspace of $\left(Y, T_{Y}\right)$ is $T_{3 \frac{1}{2}}$.

Proof. (a) implies (b): Since $\left(Y, T_{Y}\right)$ is $T_{3 \frac{1}{2}}$ and $T_{3 \frac{1}{2}}$ is a subspace property, then each subspace of $\left(Y, T_{Y}\right)$ is $T_{3 \frac{1}{2}}$. Since $\left(Y, T_{Y}\right)$ is a maximal, proper, dense, $T_{3 \frac{1}{2}}$ subspace of $(X, T)$, then, by definition, for each subset $Z$ of $X$ that properly contains $Y,\left(Z, T_{Z}\right)$ is "not- $T_{3 \frac{1}{2}}$ ". 
(b) implies (a): Since $Y$ is a subspace of itself, then $\left(Y, T_{Y}\right)$ is a proper, $T_{3 \frac{1}{2}}$ subspace of $(X, T)$ and since for each subset $Z$ of $X$ that properly contains $Y,\left(Z, T_{Z}\right)$ is "not- $T_{3 \frac{1}{2}}$ ", then, by definition, $\left(Y, T_{Y}\right)$ is a maximal, proper, dense, $T_{3 \frac{1}{2}}$ subspace of $(X, T)$.

Definition 3.1. A space $(X, T)$ has the maximal, proper, dense, $T_{3 \frac{1}{2}}$ subspace property iff $(X, T)$ has a maximal, proper, dense, $T_{3 \frac{1}{2}}$ subspace $\left(Y, T_{Y}\right)$.

Theorem 3.2. The maximal, proper, dense, $T_{3 \frac{1}{2}}$ subspace property is a topological property.

Proof. Let $(X, T)$ have the maximal, proper, dense, $T_{3 \frac{1}{2}}$ subspace property and let $f:(X, T) \rightarrow(Z, S)$ be a homeomorphism. Let $\left(Y, T_{Y}\right)$ be a maximal, proper, dense, $T_{3 \frac{1}{2}}$ subspace of $(X, T)$. Then $f(Y)$ is a proper subset of $Z$ and since $T_{3 \frac{1}{2}}$ is a topological property and $f_{f(Y)}:\left(Y, T_{Y}\right) \rightarrow\left(f(Y), S_{f(Y)}\right)$ is a homeomorphism, then $\left(f(Y), S_{f(Y)}\right)$ is a proper, $T_{3 \frac{1}{2}}$ subspace of $(Z, S)$. Let $z \in Z \backslash f(Y)$ and let $U \in S$ such that $z \in U$. Let $x$ be the unique element of $X$ such that $f(x)=z$. Then $x \notin Y$ and $x \in f^{-1}(U) \in T$ and, since $\left(Y, T_{Y}\right)$ is dense in $(X, T)$, $f^{-1}(U) \cap Y \neq \phi$. Thus $U \cap f(Y) \neq \phi$ and $\left(f(Y), S_{f(Y)}\right)$ is dense in $(Z, S)$. Suppose there exists a subset $W$ of $Z$ that properly contains $f(Y)$ that is $T_{3 \frac{1}{2}}$. Then $f^{-1}(W)$ is a subset of $X$ that properly contains $Y$ and $\left(f^{-1}(W), T_{f^{-1}(W)}\right)$ is $T_{3 \frac{1}{2}}$, which is a contradiction. Thus for each subset $W$ of $Z$ that properly contains $f(Y),\left(W, S_{W}\right)$ is "not- $T_{3 \frac{1}{2}}$ ". Thus $(f(Y)$, $\left.S_{f(Y)}\right)$ is a maximal, proper, dense, $T_{3 \frac{1}{2}}$ subspace of $(Z, S)$. 
Theorem 3.3. The maximal, proper, dense, $T_{3 \frac{1}{2}}$ subspace property is not a subspace property.

Proof. Let $(X, T)$ have the maximal, proper, dense, $T_{3 \frac{1}{2}}$ subspace property with maximal, proper, dense, $T_{3 \frac{1}{2}}$ subspace $\left(Y, T_{Y}\right)$ having two or more elements. Let $a$ and $b$ be distinct elements of $Y$, let $Z=\{a\}$, and let $W=\{a, b\}$. Then $\left(Z, T_{Z}\right)$ is a proper, $T_{3 \frac{1}{2}}$ subspace of $\left(W, T_{W}\right)$, where $\left(W, T_{W}\right)$ is $T_{3 \frac{1}{2}}$ and "not-"not- $T_{3 \frac{1}{2}}$ '”. Thus the maximal, proper, dense, $T_{3 \frac{1}{2}}$ subspace property is not a subspace property.

The following example shows that for a space $(X, T)$, a maximal, proper, dense, $T_{3 \frac{1}{2}}$ subspace of $(X, T)$ need not be unique.

Example 3.1. Let $X=\{a, b\}$, where $a$ and $b$ are distinct, and let $T$ be the indiscrete topology on $X$. Then each singleton set subspace of $(X, T)$ is a maximal, proper, dense, $T_{3 \frac{1}{2}}$ subspace of $(X, T)$ and maximal, proper, dense, $T_{3 \frac{1}{2}}$ subspaces are not unique.

\section{References}

[1] C. Dorsett, Weakly $P$ properties, Fundamental Journal of Mathematics and Mathematical Sciences 3(1) (2015), 83-90.

[2] C. Dorsett, $T_{0}$-identification $P$ and weakly $P$ properties, Pioneer Journal of Mathematics and Mathematical Sciences 15(1) (2015), 1-8.

[3] C. Dorsett, Complete characterizations of weakly Po and related spaces and properties, Journal of Mathematical Sciences: Advances and Applications 45 (2017), 97-109.

DOI: http://dx.doi.org/10.18642/jmsaa_7100121834 
[4] C. Dorsett, Additional properties for weakly Po and related properties with an application, Journal of Mathematical Sciences: Advances and Applications 47 (2017), 53-64.

DOI: http://dx.doi.org/10.18642/jmsaa_7100121877

[5] C. Dorsett, Additional properties and characterizations of $T_{0}$ and "Not- $T_{0}$ ", and each of $R_{0}$, and $R_{1}$ in "Not-T $T_{0}$ " spaces, Pioneer Journal of Mathematics and Mathematical Sciences 21(1) (2017), 25-36.

[6] C. Dorsett, Additional properties and characterizations of $T_{0}, Q o$, weakly $Q o$ and maximal, proper, Dense, Qo OXTO subspaces in weakly $Q_{0}$ and "not- $T_{0}$ " spaces, Journal of Mathematical Sciences: Advances and Applications 49 (2018), 15-28.

DOI: http://dx.doi.org/10.18642/jmsaa_7100121916

[7] C. Dorsett, Necessary and sufficient conditions for a space to have a maximal, proper, dense, $T_{i}$ subspace, $i=1,2$, Fundamental Journal of Mathematics and Mathematical Sciences 9(1) (2018), 1-12.

[8] C. Dorsett, Infinitely many topological properties in which $T_{0}, T_{1}, T_{2}$ urysohn, $T_{3}$, and $T_{3 \frac{1}{2}}$ are equivalent and infinitely many new characterizations of the $T_{3 \frac{1}{2}}$ property, Journal of Mathematical Sciences: Advances and Applications 44 (2017), 73-89.

DOI: http://dx.doi.org/10.18642/jmsaa_7100121777

[9] M. H. Stone, Applications of Boolean algebras to topology, Matematicheskii Sbornik 1(43) (1936), 765-772.

[10] P. Urysohn, Uber die machtigkeit der zusammenhangenden mengen, Mathematische Annalen 94(1) (1925), 262-295.

DOI: https://doi.org/10.1007/BF01208659 